\title{
EL DEPORTE COMO MECANISMO DE RESILIENCIA PARA DEPORTISTAS EN SITUACIÓN DE DISCAPACIDAD ${ }^{1}$
}

\section{Sports as a Resilience Mechanism for Athletes in a Disability Situation}

\author{
Milena Gallego Agudelo ${ }^{2}$, Natalia Andrea Salinas-Arango ${ }^{3}$
}

Recibido: 2019-01-15 Aceptado: 2019-03-02

\begin{abstract}
Resumen: En este artículo resultado de investigación se evidencian experiencias de deportistas de alto rendimiento en situación de discapacidad en la ciudad de Medellín en el desde enero del 2018 hasta noviembre del 2019, que muestran la cotidianidad y la manera como el deporte es asumido por ellos como un mecanismo de resiliencia. Se realizó una investigación cualitativa, con enfoque fenomenológico, a partir de ocho historias de vida en las que se analizó la relación entre la vida personal, familiar y deportiva de los sujetos; las categorías abordadas fueron discapacidad, deporte y resiliencia. En conclusión, se presentan dificultades, retos, logros y recomendaciones de estas personas para una sociedad en tránsito a la inclusión y en la que se hace necesario un cambio de imaginarios sociales sobre la discapacidad.
\end{abstract}

Palabras clave: Discapacidad, resiliencia, deporte, redes de apoyo, historias de vida.
Abstract: In this article result of research is evidence experiences of athletes high-performance in situation of disability in Medellin from January 2018 to November 2019, showing the everyday and how the sport is assumed by them as a mechanism of resilience. Was a qualitative research, with focused phenomenological, from eight life stories in which examined the relationship between the personal life, family and sport of the subjects; the categories addressed were disability, sport and resilience. In conclusion, are presented difficulties, challenges, achievements and recommendations of these people for a society in transit to the inclusion and in which is necessary to a change of imaginary social on disability.

Keywords: Disability, resilience, sport, support networks, life stories
Para citar este artículo en APA: Gallego, M. y Salinas, N. (2019). El deporte como mecanismo de resiliencia para deportistas en situación de discapacidad. Revista de Psicología Universidad de Antioquia, 11(1), 117 135. DOI: https://doi.org/10.17533/ udea.rp.v11n1a05
Artículo derivado de la investigación "Discapacidad e inclusión social”, realizada por el Grupo de investigación en Trabajo Social (GITS) y el Semillero de investigación Dinámica Social; radicada en el Centro de investigaciones, desarrollo e innovación (CIDI) de la Universidad Pontificia Bolivariana.

2 Estudiante de Trabajo Social, Universidad Pontificia Bolivariana, Medellín, Colombia. Investigadora del Semillero Dinámica Social, adscrito al Grupo de investigación de Trabajo Social (GITS). Correo: milena.gallego@upb.edu.co; https:// orcid.org/0000-0002-6170-420X

3 Candidata a Doctora en Filosofía, Magíster en Historia, Trabajadora Social. Docente investigadora Titular de la Facultad de Trabajo Social de la Universidad Pontificia Bolivariana; https://orcid.org/0000-0003-1369-514X 


\section{Introducción}

El deporte es una actividad que tiene gran impacto en la vida humana, desde una edad temprana hasta la adultez, permitiendo el desarrollo de habilidades, actitudes y destrezas, favoreciendo distintas dimensiones del ser, como la lúdica y la biopsicosocial, desde las distintas prácticas y escenarios deportivos. Como lo menciona Alabarces "El deporte se instituye en nuestras sociedades (en el mundo) como práctica privilegiada de lo elementalmente humano, lugar donde la diferencia desaparece, el mundo se reconcilia y el conflicto cede" (1998, p. 4). En referencia al deporte, a su impacto y relación con la sociedad, Verdejo dice que,

Tradicionalmente, las Ciencias de Humanidades y Sociales han abordado el análisis del deporte como un producto cultural "de amplio espectro" que sin duda ha alcanzado enorme importancia en nuestros días. Veamos algunos ejemplos significativos de ello: el deporte se ha convertido en una de las manifestaciones humanas más importantes de los últimos decenios del siglo XX y su proyección mundial seguirá extendiéndose en las próximas generaciones no solamente como un espectáculo o afición popular, sino como objeto de ciencia (2016, p. 7).

El deporte como objeto de estudio, adquiere mayor interés investigativo, cuando los deportistas se encuentran en situación de discapacidad, dado que se ha encontrado en el deporte un mecanismo de resiliencia en su día a día. La comprensión de esta realidad, desde las experiencias e historias de vida narradas por estas personas, sugieren la indagación por los espacios deportivos, las redes de apoyo, los contextos que involucran la relación de la discapacidad y la resiliencia que se teje en torno al deporte.

A propósito de la discapacidad y el deporte, Ramírez expresa que "la discapacidad se empezó a reconocer como realidad social, desde 1948, a través de Sir Ludwig Guttman y Charlie Atkinson, Neurólogo y Fisioterapeuta respectivamente" (2010, p. 1), y a su vez parte de esta realidad, para indicar que la inclusión social comienza a formar parte de la importancia de incluir el deporte en los procesos de cambio y superación personal de los soldados afectados en tiempos de guerra. Incentivando así a la creación del Movimiento Paralímpico Universal. Para efectos de este, un gran número de países se unieron a 
este movimiento, permitiendo potencializar la inclusión en cada uno de estos países con la población que se encuentra en alguna situación de discapacidad (Ramírez, 2010).

El deporte es un generador de espacios de ocio, disciplina, integración y socialización a lo largo de la historia, en torno a este se ha construido un sistema que incluye federaciones deportivas a nivel mundial que apoyan e integran a deportistas, clubes, organizaciones no gubernamentales (ONG) y demás organizaciones. Así mismo, surge en Colombia el Comité Paralímpico Colombiano a mediados de febrero del año 2001, dando paso a federaciones que impactan en la vida de personas con alguna situación de discapacidad.

Mediante la actividad física en general como práctica recreativa o competitiva, se puede evidenciar la influencia y el impacto en cuanto al desarrollo de capacidades motrices y mentales, pero no solo en estos ámbitos, sino también en la repercusión de manera directa en los valores y aptitudes de cada persona al socializar, sentirse parte de un espacio, institución y/o colectivo. Es así como el deporte adaptado para personas en situación de discapacidad es una clara representación de cómo el deporte permite trascender, romper barreras y estigmas, generando una sociedad incluyente mediante una sana inserción de la población diversa.

Dice el medallista paralímpico Enhamed Enhamed (BBVA Aprendamos juntos, 2018) que "una de las mayores discapacidades es la falta de confianza en uno mismo, más que la ceguera o que te falte una pierna, por eso empecé a nadar, porque era mi refugio". Enhamed es un deportista español con discapacidad que compitió en natación adaptada, nació en Las Palmas de Gran Canaria en 1987, de padres saharauis, es el quinto de seis hermanos. A los ocho años quedó ciego por un desprendimiento de las córneas en sus ojos. Afrontó desde muy joven la discapacidad visual como su principal reto para salir adelante, pues afirma que, partiendo de su condición de discapacidad, aprendió que le iba a costar mucho más conseguir cada objetivo en su camino, pero se visualizó lograrlo. Enhamed Enhamed afirma:

Me propuse batir un récord del mundo y lo terminé batiendo, luego me propuse conseguir 4 medallas de oro en Pequín y al final las terminé consiguiendo y todo porque me di cuenta de que podía entrenar mi mente y visualizarme. Repasé lo 
que había sido mi vida y me di cuenta de que si no me hubiera quedado ciego no hubiera aprendido a nadar, no me hubiese esforzado como me he esforzado (BBVA Aprendamos juntos, 2018).

En este orden de ideas, la investigación partió de la pregunta ¿de qué manera el deporte es un mecanismo de resiliencia para jóvenes deportistas en situación de discapacidad de Colombia en el 2018 y 2019? Se realizó una aproximación al deporte desde una mirada incluyente, retomando así la idea de que el deporte es un mecanismo de resiliencia, reconociendo las experiencias en el deporte y en otros aspectos de la historia de vida de personas en situación de discapacidad. Por tanto, el objetivo general fue identificar la manera en que el deporte se configura como un mecanismo de resiliencia para jóvenes deportistas en esta situación en Colombia, a partir de sus experiencias deportivas en la ciudad de Medellín en el 2018- 2019.

Es por ello que la indagación por la relación entre el deporte y la resiliencia en personas con limitaciones físicas y/o mentales se muestra como un foco de superación personal, de esta manera exaltando el amor al deporte y la incidencia que este tiene en la vida de estos deportistas desde el ámbito competitivo.

De esta forma la discapacidad es una de esas circunstancias que pueden constituir una base real de formación de espacios de universalidad humana y personal ante la identidad auténtica del ser humano y su especificidad inherente. Ante ella la sociedad no puede permanecer impasible, tiene que reconocer en cada ciudadano una persona humana detentadora de derechos (Lázaro, 2013, p. 401).

Esta investigación visibilizó también por medio de las historias de vida a personas que por medio del deporte han podido superar situaciones adversas, brindando lo mejor de sí en deportes adaptados, en ámbitos competitivos o por recreación, generando de este modo una reflexión sobre qué es ser resilientes en espacios donde la inclusión y exclusión comparten el día a día. 


\section{Metodología}

Se realizó una investigación cualitativa, la cual es, según Olga Vélez y María Eumelia Galeano (2002), la misma que rescata la importancia de la subjetividad, es la garante y el vehículo a través del cual se logra el conocimiento de la realidad humana. Igualmente, el estudio se desarrolla desde un enfoque metodológico fenomenológico, al permitir tener un acercamiento e interpretación desde los sujetos de análisis mismos, desde sus propias experiencias mediante las historias de vida. La fenomenología es un enfoque que:

[...] implica el estudio en profundidad de cómo aparecen las cosas en la experiencia. La fenomenología pretende aclarar las formas de existencia humana, partiendo del ser del hombre en el mundo y proporcionar un cuadro para situar los hechos que se van contrastando (Vélez y Galeano, 2002, p. 47).

Así mismo, se resalta la importancia de emplear las historias de vida como la modalidad adecuada al momento de indagar y obtener información y/o conocimiento sobre experiencias y acontecimientos de las personas sujetos de estudio, convirtiéndose en un tipo de investigación que permite un acercamiento y una amplia comprensión de la subjetividad del ser humano en la sociedad, basados en experiencias personales.

Las historias de vida dan cuenta de anécdotas y relatos desde las personas de la experiencia, exaltando la oralidad como mecanismo para analizar la subjetividad en la realidad en determinados contextos. Para llevar a cabo esta modalidad, inicialmente se debe seleccionar un tema de interés, documentarse al respecto, conocer qué se ha investigado del mismo, y posterior a ello identificar y establecer contacto con las personas con este perfil característico.

El acercamiento a las historias de vida permite crear un ambiente de diálogo abierto, pueden existir preguntas estructuradas, pero generalmente está abierta a algunas preguntas que surgen durante la conversación puesto que la persona encargada de realizar la entrevista puede experimentar nuevos interrogantes a medida que escucha el testimonio, historia de vida, generando un flujo de conversación y ambiente de confianza, comprensión y respeto. Cabe resaltar que las experiencias de estas personas son la base y el fundamento de 
la presente investigación, porque se retoman sus experiencias deportivas partiendo de alguna situación de discapacidad.

Para efectos de la investigación, se utiliza como técnicas de recolección de información la revisión documental y las entrevistas semiestructuradas. La primera, la define Victoria Eugenia Valencia López como la que "permite identificar las investigaciones elaboradas con anterioridad, las autorías y sus discusiones; delinear el objeto de estudio; construir premisas de partida; consolidar autores para elaborar una base teórica; hacer relaciones entre trabajos" (Valencia, 2019, p. 2).

Finalmente se llevan a cabo entrevistas semiestructuradas, mediante las cuales se realizó un abordaje del objeto y sujeto de estudio de manera abierta, si bien la entrevista cuenta con varias formas para llevarse a cabo, para efectos de esta investigación, se realizan varias entrevistas semiestructuradas, las cuales tienen como objetivo obtener información de forma oral y personalizada sobre acontecimientos, experiencias, opiniones de personas, orientadas según el tema bajo el cual se desarrolla la investigación. Cabe mencionar que para Pilar Folgueiras "en la entrevista semiestructurada es esencial que el entrevistador tenga una actitud abierta y flexible para poder ir saltando de pregunta según las respuestas que se vayan dando o, inclusive, incorporar alguna nueva cuestión a partir de las respuestas dadas" (2019, p. 3).

\section{Proceso metodológico y selección de la muestra}

Para la selección de la muestra de los sujetos de análisis, se realizó una búsqueda inicial en redes sociales de personas deportistas en situación de discapacidad en la ciudad de Medellín, y, posteriormente, una primera toma de contacto para manifestar el interés de conocer sus historias de vida y experiencias deportivas. También se tuvo como referente el testimonio de un deportista profesional como informante clave, que llevó a conocer el mundo del deporte y la competencia, siendo esta una forma de iniciar la estrategia de bola de nieve, para conocer a deportistas de alto rendimiento en situación de discapacidad.

Luego del contacto se realizaron los encuentros con las personas, mediante las entrevistas semiestructuradas, de las que se conocieron sus experiencias a 
través de relatos personales, para posteriormente analizarlas desde las categorías de análisis: deporte, resiliencia, redes de apoyo y discapacidad. Simultáneo a estas entrevistas se realizó el análisis de documentales en internet de deportistas paralímpicos.

Para la realización de las entrevistas y el uso del material contenido a lo largo de los resultados, los participantes firmaron un consentimiento informado en el cual accedieron a participar en la investigación y autorizan el uso de la información suministrada para fines investigativos.

A continuación, se presenta a los sujetos estudiados, el código que se empleará para facilitar la lectura de sus testimonios, el período en el que fue realizada la entrevista, una breve descripción de su discapacidad, de aspectos generales de su vida y su participación en el deporte:

Juan Guillermo Peña Jiménez. Código JG1. Presenta discapacidad visual hereditaria de nacimiento, tiene 33 años, es profesional en masoterapia del Politécnico Jaime Isaza Cadavid, ejerce la profesión desde su hogar en el barrio el Poblado de Medellín, su núcleo familiar está conformado por su mamá, hermana y su sobrino. Deportes que practica: halterofilia y crossfiter. $^{3}$

Luis Felipe Vallejo. Código LV2. Su situación de discapacidad se basa en una lesión pregangloniar de plexo braquial (desgarre de los nervios del brazo), tiene 25 años. Es Chef egresado de la Universidad Luis Amigó, actualmente se encuentra en la mitad de carrera de profesional en deportes en el politécnico Jaime Isaza Cadavid. Vive en la ciudad de Medellín. ${ }^{4}$

Nelson Ocampo Henao. Código NO3. Su situación de discapacidad es distrofia muscular de Duchenne degenerativa. Fue director técnico de fútbol para

3 La halterofilia o levantamiento de pesas es un deporte que consiste en lograr el levantamiento del máximo peso posible de un atleta por medio de una barra en cuyos extremos se fijan varios discos, que son los que determinan el peso final que se levanta. Y el crossfit es un deporte que combina resistencia, fuerza física, flexibilidad, potencia y equilibrio. Mediante diferentes ejercicios y técnicas de ejecución.

4 El fisicoculturismo es un tipo de deporte basado generalmente en ejercicio físico intenso, en algunos casos anaeróbico, consistente - la mayoría de veces - en el levantamiento de pesas; cuyo fin suele ser la obtención de un cuerpo lo más definido, voluminoso y proporcionado muscularmente posible. Los fisicoculturistas ejecutan poses frente a un jurado, que asigna puntuaciones y otorga premios como los de míster universo. 
jóvenes y actualmente ejerce como sociólogo Egresado de la Universidad de Antioquia. ${ }^{5}$

Agustín Castro. Código AC4. Deportista Colombiano ganador de seis títulos mundiales de BMX, crossfiter y empresario. Actualmente está retirado de competencias profesionales y se desempeña como empresario de una cadena de gimnasios de crossfit en varias ciudades de Colombia, y a su vez brinda conferencias de motivación y superación personal. ${ }^{6}$

Fernando Mina Cortez, 'Tyson'. Código FM5. Entrevista en video tomada de canal Colombia Sports (2012=). Practica lanzamiento de jabalina y disco, estuvo en los Juegos Paralímpicos de Londres y medallista de los Juegos Deportivos Paranacionales 2012. ${ }^{7}$

Yanive Torres Martínez. Código YT6. Entrevista en video tomada de canal de Colombia Spots (2012). Tiene 34 años, nació en Puente Nacional, Santander, hace 10 años que vive en Bogotá, sobresalió en los Juegos Parasuramericanos de Chile 2014, al conseguir 3 medallas de oro en lanzamiento de bala, disco y jabalina.

Elkin Alonso Serna. Código EAS7. Entrevista en video tomada de canal de Colombia Sports (2012). Nació en Urrao, Antioquia, el 13 de enero de 1985. Es un atleta paralímpico consagrado, pues además de haber ganado la medalla de plata en la maratón de los Juegos Paralímpicos de Beijing 2008 y Londres 2012, llegó en el segundo lugar en el Campeonato del Mundo de Atletismo Paralímpico de Lyon, Francia, en 2013 y obtuvo la presea de bronce en los Juegos Para panamericanos $2015 .^{8}$

\footnotetext{
5 Es la persona encargada de la dirección, instrucción y entrenamiento de un deportista individual o de un colectivo de deportistas.

6 El BMX es una disciplina del ciclismo que se practica con bicicletas Cross con ruedas de 20 pulgadas de diámetro. El BMX abarca dos modalidades: carrera, cuyo objetivo es completar el recorrido en el menor tiempo posible, y estilo libre (freestyle), cuyo objetivo es realizar acrobacias.

7 El lanzamiento de jabalina es una prueba de atletismo en la que, como su nombre indica, se lanza una jabalina, hecha con material reglamentario de metal o fibra de vidrio, con el objetivo de que llegue lo más lejos posible.

8 El atletismo es considerado el deporte organizado más antiguo del mundo. Abarca numerosas disciplinas agrupadas en carreras, saltos, lanzamientos y pruebas combinadas.
} 
Enhamed Enhamed Mohamed. Código EM8. Entrevista en video tomada de canal BBVA Aprendamos juntos (2018). Es un deportista español con discapacidad visual que compitió en natación adaptada, nació en Las Palmas de Gran Canaria en 1987, de padres saharauis es el quinto de seis hermanos. ${ }^{9}$

\section{Resultados}

\section{Personas en situación de discapacidad y su relación con el deporte}

Partiendo de dos realidades cotidianas que comparten los deportistas entrevistados, las cuales son la práctica deportiva a nivel de competencia y alguna limitación física o motriz, con el acercamiento a sus historias de vidas, se encontró que la situación de discapacidad, en cierto punto de sus vidas, se configuró en un motor que los impulsó al logro de objetivos y sus sueños por medio del deporte; hasta llegar a retarse a sí mismos con grandes metas deportivas con pares, donde cada uno entrega lo mejor de sí y no hay barreras en su camino.

La historia de vida de cada uno de ellos evidencia que la discapacidad marca una pauta trascendental, es una muestra de cómo se vuelven sujetos resilientes a partir del campo de los deportes, que les da elementos también de interpretación de su propia realidad y de su vivencia cotidiana.

Por ejemplo, cuando YT6 cae de una gran altura de un árbol, al despertar queda en condición de discapacidad en silla de ruedas y mientras está en rehabilitación y en el proceso de aprender a manejar la silla, se encuentra en la ciudad de Bogotá con el que actualmente es su entrenador físico, quien le da la oportunidad de comenzar a entrenar. Afirma YT6 que "ese primer día de entrenamiento recuerdo mucho que llovió demasiado, y fue pues bonito, era un deporte nuevo para mí, y ese mismo día vi la capacidad en mí, yo me inspiro cuando entreno".

LV2 sufrió un accidente automovilístico generando una lesión preganglionar de plexo braquial (desgarre de los nervios del brazo) fractura de húmero,

\footnotetext{
- La natación es el movimiento y el desplazamiento a través del agua mediante el uso de las extremidades corporales y por lo general sin utilizar ningún instrumento o apoyo para avanzar.
}

DOI: https://doi.org/10.17533/udea.rp.v11n1a05 
clavícula y escápula. LV2 desde muy niño tuvo el deporte como fuente de educación, afirma que el deporte significa todo para él, pues su rutina diaria se basaba en entrenar muy temprano, trabajar como entrenador y estudiar, todo gira en torno a esto.

El sociólogo francés Marcel Mauss (1979) consideraba el deporte como un "hecho social total" por su capacidad de funcionamiento como un sistema social completo capaz de involucrar a los diversos ámbitos de la sociedad. La sociedad también cuenta con representaciones sociales frente a la discapacidad, que pueden generar procesos de exclusión (Soto y Vasco, 2008).

\section{Redes de apoyo e impacto en la vida de los deportistas}

Las redes de apoyo son comprendidas como aquellas relaciones mediante las cuales los individuos se vinculan de una u otra manera a otros individuos, grupos o instituciones que los rodean, conformando dinámicas relacionales particulares dentro de una sociedad. Algunos autores afirman que:

Una red social es el campo relacional total de una persona y tiene por lo común una representación espaciotemporal. Su grado de visibilidad es bajo, pero en cambio posee numerosas propiedades vinculadas con el intercambio de información. Tiene pocas reglas formales, pero está compuesta por las relaciones entre muchas personas, algunas de las cuales son conocidas por muchas personas, algunas de las cuales son conocidas por muchas personas entre ellas, siendo a menudo este nexo, ignorado por las personas conectadas. (Speck y Attneave, 1973, citado por Lomnitz, 1981, p. 42)

Si bien la familia, como institución primaria en la cual se aprenden normas, cultura y valores para formar nuestra personalidad, es la primera y principal red de apoyo, en el trascurso de la vida la generación de lazos afectivos hace que se amplíen estas redes a las relaciones de amistad, trabajo y apoyo profesional. Tal como lo menciona AC4, quien indica que, en cuanto a sus redes de apoyo a nivel personal y en su carrera como deportista, cuenta con un entrenador mental y un psicólogo que le brindan una claridad emocional, que de la mano consigo mismo, son el soporte para la automotivación necesaria para seguir adelante. A lo largo de su vida deportiva ha sentido mucha inspira- 
ción en los deportistas paralímpicos, pues según indica, las limitaciones están en la mente y no en el cuerpo físico.

Así mismo, Mony Elkaim (citado en Chadi, 2000) plantea que la red social es un grupo de personas, miembros de una familia, vecinos, amigos y otras personas, capaces de aportar con una ayuda y un apoyo tan reales como duraderos a un individuo o una familia. Chadi (2000) indica que las redes sociales se clasifican en primarias, secundarias y terciarias. Las primeras componen la familia, amigos y vecindario; las segundas como relaciones laborales o de estudio y las últimas son las redes institucionales.

En relación con el impacto que genera el entorno y/o las redes de apoyo, $\mathrm{NO} 3$ indica que siempre estuvo rodeado de un ambiente sobreprotector por parte de su familia, los cuales buscaban que no se lastimara. Sin embargo, la sobreprotección familiar a raíz de sus limitaciones físicas, no frustraron sus aspiraciones pese al temor de que se expusiera a un medio hostil, por ejemplo, a algo tan básico como la estructura física de la universidad en la que estudió, dado su desplazamiento en la silla de ruedas. Sin embargo, esta ni otras situaciones limitantes, fueron obstáculo para que él encontrara la colaboración de otras personas para poder acceder a ciertos lugares, en este caso las aulas de clase, acceso que no se encontraba adaptado para personas con movilidad reducida; NO3 siempre encontró apoyo para vencer obstáculos de su día a día.

Radcliffe-Brown (citado en Izquieta, 1996) afirma que las relaciones familiares surgen a través de la procreación (vínculos de sangre) y habitualmente, aunque no necesariamente, mediante el matrimonio (vínculos de afinidad). Dentro de la red familiar sobresale, por su intensidad, la relación madre-hijo, unidad elemental e irreductible. Por medio de este vínculo el niño aprende a crear lazos con los demás, y construye autoconfianza (Izquieta, 1996).

Con relación a los vínculos y/o cuidados que se pueden recibir dentro de un hogar por parte de los progenitores o con quienes se convive, EM8 afirma que:

La sobreprotección mata a las personas, en cuanto a que no se les permite salir al mundo a explorar por sí mismos la realidad a la cual se deben enfrentar, pues en su interés por protegerle de los peligros, pueden llegar a aislar a aquellos que necesitan conocerse y desenvolverse en el medio, mostrando sus capacidades de 
valerse por sí mismo hasta donde sus limitaciones físicas se lo permitan. (BBVA Aprendamos juntos, 2018).

\section{Discapacidad y resiliencia}

Históricamente se han utilizado diversos conceptos para referirse a la discapacidad, a veces incluso como sinónimos. Los términos enajenado, retrasado, incapaz, inválido, deficiente, minusválido o discapacitado se repiten en los textos normativos tanto nacionales como internacionales y no siempre para reflejar la misma realidad. Esta confusión no es una mera cuestión terminológica, sino que, como advierte (Verdugo, citado por Biel, 2009), sobre estas expresiones subyacen una serie de valores y actitudes determinados (Biel, 2009, p. 19). Según la Organización Mundial de la Salud:

\footnotetext{
Discapacidad es un término general que abarca las deficiencias, las limitaciones de la actividad y las restricciones de la participación. Las deficiencias son problemas que afectan a una estructura o función corporal; las limitaciones de la actividad son dificultades para ejecutar acciones o tareas, y las restricciones de la participación son problemas para participar en situaciones vitales. Por consiguiente, la discapacidad es un fenómeno complejo que refleja una interacción entre las características del organismo humano y las características de la sociedad en la que vive. (Organización Mundial de la Salud, 2019)
}

El término resiliencia al igual que muchos otros términos, se han dado a la tarea de intentar dar la definición más clara posible y por ende la dificultad de elegir una como la más opcional y general que se acomode a cualquier situación, si bien no hay alguna definitiva, si se plantea algunas como las más adecuadas a tratar. Por ejemplo, Garmezy (1991; citado por Becońa, 2006) la define como la capacidad para recuperarse y mantener una conducta adaptativa después del abandono o la incapacidad inicial de iniciarse un evento estresante (p. 459).

Para LV2 un evento estresante que lo sacó de su 'zona de confort', fue aquel día en el cual sufrió un accidente automovilístico, posterior a ello creía que su vida iba a cambiar, pero su principal motivación es el deporte, seguir ganando títulos deportivos con el fisicoculturismo, y con total convicción afirma LV2 "Yo creo que me puse una meta muy alta, pero las limitaciones las tiene la 
mente y el deporte es el mejor aliado, si vos tenés disciplina y te refugias en el deporte vos avanzas demasiado, no solo físicamente sino en otros aspectos". Permitiendo que todas las barreras, que tanto con veredictos médicos y opiniones del mundo en el que se desenvuelve, pasen a un segundo plano, la convicción que todo lo que se proponga va a trascender por medio de su profesión como deportista, es inquebrantable para LV2.

En relación con lo anterior, Masten (2001; citado por Becoña, 2006) ha definido la resiliencia como un tipo de fenómeno caracterizado por buenos resultados a pesar de las serias amenazas para la adaptación o el desarrollo (p. 228). Más adelante Masten y Powell señalan que la resiliencia implica "patrones de adaptación positiva en el contexto de riesgo o adversidades significativas" (2003, p. 4; citado por Becoña, 2006, p. 128). Para ambos autores, la resiliencia debe concebirse como la descripción de un patrón general más que un diagnóstico, por esto, es más apropiado decir "esta persona tiene un patrón resiliente" o "esta persona muestra las características de la resiliencia". También concuerdan y concluyen estos autores que la resiliencia no debe ser vista como el rasgo de un individuo, debido a la variación que presenta según el tiempo, la etapa de vida, conductas, entre otras.

Asegura EM8: "Si no hubiera sido ciego, seguramente no hubiera sido tan feliz", pues su actitud frente a la discapacidad, que afronta desde muy pequeño, hizo que su voluntad más férrea para esforzarse a realizar mucho mejor las actividades, tanto cotidianas como deportivas desde que emprendió su carrera como nadador profesional en España, logró, más que demostrarle al mundo, demostrarse a sí mismo cuál era su capacidad de luchar por sus ideales y que, en medio de su constante aceptación, la natación fue su aliada para ser quien es en estos momentos y lograr destacarse como uno de los mejores nadadores paralímpicos del mundo.

El concepto de resiliencia no implica tanto una invulnerabilidad al estrés sino la habilidad de recuperarse de eventos negativos (Garmezy, 1991). Así, Fonagy et al., describen la resiliencia como "el desarrollo normal bajo condiciones difíciles" (p. 128) y Masten (2001) como "un fenómeno común que surge de los procesos adaptativos humanos ordinarios" (p. 234). Para su estudio, uno de los mayores problemas es que no existe una teoría unificada de la resiliencia que permita guiar claramente a qué nos referimos y qué podemos hacer para potenciarla (Luthar, 
Cicchetti y Becker, 2000), aunque se han dado pasos importantes en este sentido en los últimos años (ej., Kumpfer et al., 1998) (Becońa, 2006, p. 128).

De esta manera, el deporte puede verse como un mecanismo para ser resiliente ante las adversidades; en este sentido, LV2 afirma: "Pasé muchos momentos complejos en mi ritmo de vida, pero con la disciplina, la exigencia que representa competir en este gremio, siempre tuve la convicción y la seguridad de que iba a ser capaz" y es ese mismo pensamiento el que lo mantiene firme ante sus convicciones de estar nuevamente logrando conseguir grandes trofeos y triunfos.

Bien afirma Quińones (2006; citado por Salgado, 2012) que "el concepto de resiliencia posibilita una nueva valoración y reconocimiento en cuanto a la representación y potenciación de lo humano" (p. 130). De este modo, para LV2 el deporte es su principal herramienta de resiliencia, para demostrarse a sí mismo cuán capaz es de no limitarse, pues deja muy claro que ningún veredicto médico opaca sus proyecciones y deseos para retomar su ritmo de vida y competir.

Por tanto, es evidente que "La resiliencia se produce como consecuencia de procesos ordinarios, es decir, no se basa en cualidades especiales o extraordinarias" (Carretero, 2010, p. 70; citado por Salgado, 2012); es así como una persona llega a ser resiliente cuando ha vivido la adversidad y construye significados y modos de superarla, además de cómo se reconstruye a partir del dolor, esto según Salgado (2012).

Por ejemplo, en relación a la capacidad de resiliencia que afronta desde muy joven, NO3 comenta como sus limitaciones físicas no fueron impedimentos para soñar y proyectarse en grande, y desde que veía rodar un balón de fútbol en las calles quería jugar con sus amigos del barrio, pero su enfermedad comienza a ser un limitante para jugar con ellos, pero él tenía una convicción y pasión por el fútbol, la misma que no iba a morir ahí, pues NO3 estudia y se prepara por cuenta propia en todo lo relacionado a la dirección de un equipo de fútbol, teniendo todas las bases teóricas para poner en práctica, éste comienza muy joven a dirigir equipos de fútbol, en los que como bien menciona "no creían que una persona con discapacidad física en avanzada 
etapa , pudiera dirigir justo un equipo de fútbol”. Logrando esta meta, NO3 lleva a estos equipos a grandes competencias y se destaca en el fútbol, siendo el deporte el primer trampolín para romper barreras y no dejarse opacar por críticas o estigmas sociales.

Con relación al anterior testimonio de NO3, es necesario entonces, descubrir la realidad única y singular que vive cada persona cuando enfrenta la adversidad, sus recursos, fortalezas, riquezas, pero también sus debilidades, conflictos, ansiedades, creencias, en otros sentimientos, todo aquello que le da un carácter particular al modo cómo enfrenta el dolor y el sufrimiento. Además, es necesario conocer las redes sociales y de apoyo de la persona y su grupo familiar y demás aspectos que puedan influir en ella, se debe tener en cuenta cómo percibe esta persona o familia la adversidad y su reacción ante ella y así identificar qué impacto le genera. Es una capacidad universal, es decir, toda persona tiene el potencial de desarrollar rasgos y conductas resilientes, independientemente de su edad, sexo, grupo étnico, religión, factores sociales, políticos, económicos, culturales y/o educativos (Salgado, 2012).

Si bien una persona puede encontrar la capacidad de ser resiliente en algunos campos en los cuales se desempeña, en este caso se evidencia, cómo en relación con el deporte, la resiliencia es un valor agregado a la vida de las personas en situación de discapacidad. JG1 afirma que "sin este deporte no fuera nada de mi vida, el deporte es amor, paz adrenalina, motivación, no hay que dejarse caer por nada en el mundo", asegurando, así mismo, que su mamá es un gran apoyo en el deporte que practica y toda su familia.

La resiliencia grupal es la combinación de una capacidad innata de resistir la destrucción, tanto en personas individuales como en sistemas sociales, complementada por la habilidad de aprender de lo sucedido y buscar, conjuntamente, como convertir la crisis en oportunidad para el crecimiento. Es una serie de procesos sociales e intrapsíquicos que posibilitan tener una vida sana viviendo en un medio insano (Brenson, 2019, p. 1).

Para EAS7 "el entrenamiento debe ser tan duro que la competencia sea un paseo. Visualizarse donde se quiere estar y luchar para conseguirlo, no dejar pasar entrenamientos y dar lo mejor de sí mismo”. Así mismo EM8 afirma "No creo en los sueños, yo tengo planes" en relación con proyectarse, y luchar 
por lo que se desea alcanzar, más que un sueño asegura que se trata de tener un plan a seguir, aferrarse a él y no desfallecer hasta conseguirlo.

\begin{abstract}
Busque oportunidades para descubrirse a sí mismo, muchas veces como resultado de su lucha contra la adversidad, las personas pueden aprender algo sobre sí mismas y sentir que han crecido de alguna forma a nivel personal. Enfrente los problemas. Ante situaciones adversas tome acciones decisivas, es mejor que ignorar los problemas y las tensiones, y desear que desaparezcan (American Psychological Association, 2019).
\end{abstract}

La constancia y la decisión son claves para permanecer en ese nivel FM5 asegura que "se debe ser constante y no desfallecer, pues si tu cuerpo no da la talla no vas a poder rendir en tu competencia”. Así mismo, AC4 asemeja el deporte con la vida cotidiana, tiene un dicho que aplica y es "muéstrame a una persona entrenar o hacer deporte y te digo qué tipo de persona es, y no es precisamente cuán rápido corra, cuánto peso levante, es la actitud con la que entrena una persona, con la que hace deporte que permite hablar del tipo de persona que es, asegura que el deporte brinda esa fuerza de voluntad, fortaleza mental que tanto se necesita en el día a día”.

\title{
Discusión y conclusiones
}

El deporte y la sociedad en todos los niveles se transforman, permitiendo que no solo se base en la enseńanza y/o práctica de este, sino que se encuentra permeado por contextos sociales, políticos, económicos, facultando así que se replantee la idea de una sociedad más incluyente.

Una condición necesaria para lograr un desempeño óptimo en las prácticas deportivas de los jóvenes en situación de discapacidad, es poder contar con espacios que no se limiten a actividades lúdicas de inclusión, sino donde estos puedan desarrollar su potencial deportivo, en los que se hallen medios adaptados para poder tener una experiencia placentera y motivadora para la consecución de sus objetivos en la vida deportiva.

La práctica del deporte no implica solo fines físicos o de desarrollo de destrezas, sino que también involucra capacidades y habilidades para desenvolverse en el medio social como lo son la disciplina, la convivencia y el trabajo 
en equipo. Por tanto, en este ámbito deportivo es fundamental la dimensión humana como forma de vida en la cotidianidad de la persona en situación de discapacidad.

En este orden de ideas, se considera que el deporte como forma de resiliencia debe tener mayor incidencia para los jóvenes en el medio; cada deportista brinda lo mejor de sí y sobresale a pesar de las adversidades que puedan existir y fomenta a su vez un trabajo en equipo sano y los valores ciudadanos.

Los jóvenes consultados identifican que aún los espacios deportivos no están adaptados a sus necesidades deportivas. En este contexto, es necesario analizar en qué medida la resiliencia por medio del deporte en estos jóvenes se ve interrumpida por espacios poco adaptados en la ciudad, en su totalidad, para la movilidad de las personas bajo alguna situación de discapacidad. De esta forma, es un imperativo que las políticas públicas relacionadas con la inclusión tengan en cuenta la discapacidad y a los deportistas en la ciudad de Medellín.

Finalmente, para estos deportistas la situación de discapacidad no es un límite, este se encuentra como dificultad adicional por factores externos, más que por sus prácticas deportivas como tal, pues al fijarse una meta e intentar llevarla a cabo se encuentra en ellos una convicción inquebrantable. El deporte se ha convertido para estos jóvenes en su mayor mecanismo para sobresalir, expandir fronteras y romper con imaginarios sociales de imposibilidad frente a la vida y a los desafíos que con ella pueden llegar día a día.

\section{Referencias}

Alabarces, P. (1998). ¿De qué hablamos cuando hablamos de deporte? Nueva Sociedad, (154), 74-86. Recuperado de https://nuso.org/media/articles/downloads/2669_1.pdf

American Psychological Association. (2019). Camino a la resiliencia. Recuperado de https://www.apa.org/centrodeapoyo/resiliencia-camino

BBVA Aprendamos Juntos. (9 de abril de 2018). Cómo convertirnos en la persona que queremos ser [Archivo de video] Recuperado de https://www.youtube.com/ watch?v=x7jrGIsIQUA

Becońa, Е. (2006). Resiliencia: definición, características y utilidad del concepto. Re- 
vista de Psicopatología y Psicología Clínica, Vol. 11 (3), pp. 125 - 146. Recuperado de http://aepcp.net/arc/01.2006(3).Becona.pdf

Biel, I. (2009). Los Derechos de las personas con discapacidad en el marco jurídico internacional universal y europeo. (Tesis doctoral). Espańa: Universitat Jaume I. Recuperado de https://www.tdx.cat/bitstream/handle/10803/384628/Tesis_2010_ biel_israel_derechos\%20personas.pdf?sequence=1\&isAllowed=y

Brenson, G. (2019). La facilitación de resiliencia grupal. Amauta Internacional, 1-5. Recuperado de http://www.imaginar.org/div/facilitar/docs/amauta/RESILIENCIA-ARG.pdf

Colombia Sports. (23 de agosto de 2012). Sueños sin Limite [Archivo de video] Recuperado de https://www.youtube.com/watch?v=aBNzJdWFqMQ

Chadi, M. (2000). Redes sociales en el Trabajo Social. Buenos Aires: Espacio editorial.

Folgueiras, P. (2019). La entrevista. Documento de trabajo. Recuperado el día 15 de enero de 2019 de diposit.ub.edu: http://diposit.ub.edu/dspace/bitstream/2445/99003/1/entrevista\%20pf.pdf

Izquieta, J. (1996). Protección y ayuda mutua en las relaciones familiares. Tendencias y retos actuales. Revista Española de investigaciones sociológicas, 94(96), 189-208.

Lázaro, M. (2013). La discapacidad: una oportunidad de humanidad. Carthaginensia, XXIX, 395-418.

Lomnitz, L. (1981). Cómo sobreviven los marginados. México: Siglo XXI.

Organización Mundial de la Salud. (2019). Recuperado de https://www.who.int/topics/disabilities/es/

Ramírez, P. (2010). Desarrollo paralímpico colombiano. Revista digital, (145), 1-1. Recuperado de https://www.efdeportes.com/efd145/desarrollo-paralimpico-colombiano.htm

Salgado, A. (2012). Efectos del bienestar espiritual sobre la resiliencia en estudiantes universitarios de argentina, Bolivia, Perú y república dominicana. (Tesis de Grado). Lima: Universidad Nacional Mayor de San Marcos. Recuperado de http://cybertesis.unmsm.edu.pe/bitstream/handle/cybertesis/3293/Salgado_la.pdf?sequence $=1$ \&isAllowed $=y$

Soto, N., y Vasco, C. (2008). Representaciones sociales y discapacidad. Hologramática, 5(8), 3-22. Recuperado de http://www.cienciared.com.ar/ra/usr/3/568/ hologramatica08_v1pp3_23.pdf

Valencia, V. (2019). Revisión documental en el proceso de investigación. Universidad 
El deporte como mecanismo de resiliencia para deportistas en situación de discapacidad

Tecnológica de Pereira. Recuperado de https://univirtual.utp.edu.co/pandora/ recursos/1000/1771/1771.pdf

Vélez, O., y Galeano, M. (2002). Investigación Cualitativa Estado del arte. Medellín: Universidad de Antioquia. Recuperado de http://josemramon.com.ar/wp-content/uploads/Estado-Del-Arte-Sobre-Fuentes-Documentales-en-Investig-Cualitativa.pdf

Verdejo, D. (2016). Deporte: cultura y contracultura. Un estudio a través del modelo de los horizontes deportivos culturales. Ciencias Aplicadas, (67), 6-16. Recuperado de https://docplayer.es/36346595-Deporte-cultura-y-contracultura.html 